\title{
Pascale Bourgain
}

\section{La dramatisation de l'histoire biblique dans la poésie carolingienne}

Quand on parle de poésie biblique carolingienne, on ne peut partir évidemment que du superbe livre de Francesco Stella, et de ses autres travaux. ${ }^{1}$ Ajouter quelque chose à ce monument tiendrait de l'exploit. Pourtant, ayant récemment travaillé sur le même sujet aux alentours du XII ${ }^{\mathrm{e}}$ siècle, et ayant exploré, dans le cadre de l'entreprise menée par Fr. Stella sur les rythmes, ${ }^{2}$ les manuscrits carolingiens, j'ai été tentée de passer quelques temps dans l'intimité de ces textes. La manière dont les copistes réagissent face aux discours directs m'avait spécialement intéressée, à propos notamment des Versus de Lazaro de Paulin d'Aquilée, ${ }^{3}$ que Christiane Cosme a prévu de vous présenter, ce qui montre bien que c'est un des sommets de la poésie de l'époque. C'est ce qui m'a poussée à m'engager sur ce thème incommode, plutôt pour le plaisir que dans l'espoir d'ajouter un arbrisseau à la forêt.

Thème incommode et sûrement mal formulé. Que veut dire en effet la dramatisation ? Le propre de la poésie est de magnifier et de concentrer le sens. On peut attendre un accent mis sur la célébration de choses ou de situations, et parler alors de lyricisation, ou d'accent mis sur les faits, transmis par une narration, et parler d'épicisation, les deux catégories n'étant pas étanches. Dans le cas de la Bible, la prose de la Vulgate véhicule déjà une qualité poétique interne. En découle une poésie de célébration, méditation, prière, prenant pour matériau les livres sapientiaux et le Cantique ; ou une poésie plus narrative, prenant appui sur les livres historiques, et y ajoutant la forme épique la plus propre, pour les auditeurs et lecteurs, à faire percevoir la grandeur et l'importance des faits narrés.

A première vue, ce serait cette direction, celle de la paraphrase de textes narratifs, qui serait plus propre à la dramatisation. Mais de fait c'est l'ensemble de la Bible, qui est le grand code historique du salut de l'homme, où s'inscrit le drame, drame général de l'humanité et personnel de chaque être humain. Or, par l'intégration des éclaircissements exégétiques, la présence permanente de ce caractère dramatique affleure dans presque toutes les réalisations poétiques, sauf dans ce qu'on peut appeler 'paraphrase pure'. On n’oublie pas, en célébrant les épisodes bibliques, que leur aboutissement est toujours le même : le salut du genre humain, sa libération des incertitudes de sa condition mortelle et du péché. Ainsi l'horizon du

1 Stella (1993) ; Stella (1995); Stella (2001); Stella (2010).

2 Poesia (2000) ; Poetry (2003) ; Corpus (2007).

3 Voir Bourgain (2016) 149-181, notamment pl. 22 et 26. Paulin pratique les enjambements, ce qui est rare en poésie rythmique, et a beaucoup de discours directs avec des changements rapides de locuteur : cette souplesse prend les copistes au dépourvu. 
salut, «en qui trouve sens tout effort intellectuel» ${ }^{4}$ est quelque chose de dramatique parce qu'il implique profondément tant la communauté humaine que chaque âme, donc collectivement et individuellement (ce qui implique en gros deux sens allégoriques, l'anagogie et la tropologie). L'angoisse existentielle de l'attente du jugement, donc l'eschatologie, irrigue la poésie pénitentielle, abondamment représentée, avec la lamentation récurrente «malheureux, que ferai-je au jour du jugement ?». Dans nos textes, même s'ils ont un point de départ narratif, le salut ou le jugement apparaît presque toujours à la fin du poème, ou en filigrane sous la présentation, ou dans les refrains, dont la répétition exacerbe l'angoisse. C'est le jugement futur qui oriente et donne son sens au début du poème. Ceci est spécialement sensible dans les poèmes rythmiques, qui sont généralement antérieurs aux réélaborations plus sereines de la renovatio carolingienne. ${ }^{5}$

Quelques exemples parmi des rythmes alphabétiques, dont on ne peut donc pas croire qu'ils sont incomplets ; les rubriques font généralement référence au début du poème plus qu'à sont sujet véritable :

- Un rythme 'De adventu Domini', refrain Venite et gaudete nato Christo Domino va jusqu'aux noces de Cana, puis passe à la Résurrection et à la descente aux Enfers. ${ }^{6}$ La mention de l'Incarnation, premier Avent, semble bien avoir appelé l'idée du second Avent. Aussi l'insistance sur le retour du Christ pour le jugement est-elle un thème récurrent.

- $\quad$ Le rythme 8, 'De nativitate Christi', Alta prolis sanctissime, saute du choix des apôtres à l'ensevelissement par Joseph d'Arimathie et à la Résurrection, la dernière strophe (Zelum <tunc Gestas> perdidit) étant pour le mauvais larron. ${ }^{7}$ Même la jeunesse du Christ, ses 'Enfances' dirait-on en français, ne se comprend que dans l'optique de sa Passion. La prédication du Christ et ses miracles ne reçoivent pas la même attention.

4 Stella (1993) 48. La plupart des pièces qui seront citées sont accessibles dans les Poetae latini aevi carolini (PLAC) des Monumenta Germaniae historica. Ceux qui ont conservé une notation musicale sont réédités dans Corpus (2007).

5 PLAC IV, r. 13, 17, 23, 54.

6 A superna celi parte, PLAC IV 477-480, r. 7. Autre poème centré uniquement sur l'Avent : Qui de morte estis redempti, in Corpus p. 353. Celui-ci est tragique par son sujet, mais remarquablement mesuré et compact dans sa forme. (Il reprend le Dies irae dies illa de Sophonie 1, 15). Autres manifestations de cette centralité du thème eschatologique dans les poèmes rythmiques : Adorate omnes gentes ... Refrain Grandis pavor in mundo erit / quando deus iratus venit, PLAC VI 227, et une autre addition du même manuscrit Paris lat. 242, ibid. 230, Audite vobis omnes / quod erit a iudicio. La très grande fréquence des poèmes pénitentiels va dans le même sens ; la crainte du jugement y est parfois explicite, comme en PLAC IV 489, r. 12, 'De commendatione uniuscuiusque animae', str. 5 : Ecce in illa die iudicii / qualis ego ero miser / qui tanta peccata feci ! ; r. 14, p. 495, 'Versus de contemptu mundi', str. 11 : Luge modo, dum tempus est, / ne gemas in iudicio, et str. 19-20 ; r. 18, p. 510, 'De accusatione facinoris', str. 15, 2 : Miser ego quid faciam in die iudicii, etc.

7 Ce qui suppose une trace de l'évangile de Nicodème qui cite leurs noms, comme la str. 18 sur l'ouverture des enfers et l'évasion d'Adam. PLAC IV 480. 
- Les rythmes 13 et 17 insistent sur le Jugement. Le second et plus ancien, Apparebit repentina, titré 'De die judicii', utilise beaucoup les prophètes et les évangiles, avec des adaptations facilitées par la forme rythmique. ${ }^{8}$ Le premier, non alphabétique, Apparebit ante summum, s'intitule 'De Enoch et Haeliae', mais c'est une suite de prophéties prises autant dans les épîtres et les évangiles que dans les prophètes ou l'Apocalypse, avec pour refrain Imminente die judicii, puis In pavendo die judicii.

- L'un des plus répandus de ces rythmes, Gratuletur omnis caro, ${ }^{9}$ intitulé 'De nativitate Domini', commence bien à la Nativité, (deux strophes), continue avec le baptême (deux strophes), les miracles (deux strophes), la Passion (deux strophes), puis (str. 9-13) s'étend longuement sur la descente aux Enfers (qui figure dans le Credo, probablement d'après la même élaboration que ce qui préside à la rédaction de l'Évangile de Nicodème, mais pas dans les Évangiles) pour annoncer le Jugement. La présence dominante du jugement, individuel ou particulier, dans les poèmes rythmiques qui nous sont parvenus, est en soi un élément de dramatisation.

C'est sans doute pour cela aussi qu'une grande partie de ces textes, les plus anciens semble-t-il, et surtout les textes rythmiques où la forme strophique appelle à morceler les épisodes, sont assez indifférents à la succession dans le temps des épisodes bibliques. Le récit, notamment dans les résumés et autres microformes, selon l'expression de F. Stella, peut être tout à fait dans le désordre, car l'intérêt est de toute façon actuel. Ce sont des vignettes, qui toutes préfigurent l'Incarnation ou l'accompagnent, leur succession a moins d'importance que leur unique direction qui pointe vers la Résurrection. Le rythme Ama puer castitatem $^{10}$ est une méditation qui n'est absolument pas portée par le déroulement historique : sont évoqués dans l'ordre Jugement, incarnation, miracles, jugement, baptême, création, multiplication des pains, bâton de Moïse, miracle de la femme hémorroïde, Noé, jugement des Juifs, Pierre marchant sur les eaux... Il est imité par le r. $n^{\circ}$ 41, dans l'ordre : Annonciation, multiplication des pains, incarnation, appel aux apôtres après la Résurrection, Hérode et fuite en Egypte. Et cela arrive aussi dans des rythmes qui ne sont pas alphabétiques, ce qui fait qu'on ne peut pas incriminer le désordre alphabétique pour cette indifférence à la chronologie ; ainsi le rythme Alpha et omega ${ }^{11}$, 'De nominibus sanctorum', est un résumé biblique sous forme de portraits, avec une suite Jésus, Adam, Abel, Enoch, Noé, etc. Il s’agit de signification, pas de récit, et l'histoire ne se comprend que transmutée en son aspect de vérité éternellement prédestinée.

\footnotetext{
8 PLAC IV 491 et 507.

9 PLAC II $252 \mathrm{n}^{\circ} 13$; Corpus (2007) 251.

10 PLAC IV 573-575, n XLVIII.

$11 \mathrm{~N}^{\circ}$ 52, PLAC IV 630.
} 
Si l'on passe aux grands auteurs carolingiens, Florus de Lyon concentre toujours son propos sur le but de l'Incarnation qui est le salut. ${ }^{12}$ Il insiste très peu sur les éléments émotifs, ne joue jamais avec un pathétique facile, ne s'apitoie pas sur les souffrances de la Passion : il suit une veine très intellectuelle, qui fait penser, beaucoup plus tard, à Maître Eckart et à sa mystique toute cérébrale. Lorsque Florus paraphrase Daniel et les trois enfants dans la fournaise, ${ }^{13}$ c'est l'hymne qui l'intéresse, la position douloureuse des chanteurs n'est mentionnée qu'en passant. Il existe aussi toute une veine, de paraphrase pure, ou de paraphrase qui insiste plus sur le dogme que sur le pathétique, qui ne conserve de la valeur dramatique de la Bible qu'un profond sentiment d'appartenance et d'actualité du message. Toute la partie de résumés didactiques, les tituli, une grande partie des hymnes, qui sont célébration essentiellement, ainsi que les œuvres d'auteurs sereins comme Alcuin ${ }^{14}$ ou Florus, se passent fort bien de toute dramatisation extérieure à la conscience de cet enjeu du salut. Vivant sub gratia, après l'Incarnation, ils ont conscience de vivre dans la lumière de la grâce, qui a rendu le salut possible, et les épisodes de l'histoire biblique ne font que leur redire la même histoire prévue depuis toujours. Ils la méditent et la magnifient, mais les auteurs les plus conscients, comme Théodulfe d'Orléans à propos du bonheur des méchants chez Jérémie ou Job, ${ }^{15}$ n'éprouvent pas le besoin d'ajouter de l'emphase ou du pathos à leur reformulation poétique.

Mais le drame, dans nos esprits, a aussi d'autres connotations, plus techniques. Il représente un mode de représentation pathétique, fondé sur des procédés visant à susciter l'émotion, avec une dose de théâtralité : interjections, apostrophes, dialogues, discours directs. Ce sont ces marques, qui font partie de l'arsenal rhétorique, dont il nous faut évaluer l'emploi. ${ }^{16}$

Parce que c'est le but de la rhétorique, ses procédés les plus naturellement employés amènent à rendre l'expression pathétique et proche, à y impliquer l'auditeur. La répétition, quasi litanique et lancinante, fait la force des pièces à refrain, généralement rythmiques. Elles rapprochent ces pièces du genre de la prière, avec répons de l'assistance, ce qui est une des possibilités d'emploi du refrain (et ces refrains sont effectivement souvent une imploration, comme dans Ad te Deus ${ }^{17}$ ). Parmi les différents procédés d'emphase, Francesco Stella a insisté sur les apostrophes, ${ }^{18}$ les interpellations : ce sont des moyens de rapprocher une narration de l'auditeur, par l'utilisation des premières et secondes personnes, en dynamisant le récit.

12 Stella (1993) 212-220, et Stella (1995) 36.

13 PLAC II 374 ; Stella (1993) 247.

14 Voir $\mathrm{n}^{\circ}$ 69, PLAC I 288.

15 PLAC I 463.

16 Je ne ferai pas de différence entre les formes concentrées ou au contraire développées de la paraphrase, la typologie de Francesco Stella ayant parfaitement balisé le terrain.

17 PLAC IV 602.

18 Stella (1993) 203. 
L'influence du genre de la prière et l'habitude de s'adresser à la divinité facilitent l'emploi de la deuxième personne, même à propos d'épisodes bibliques, avec en contrepartie soit le 'nous' collectif des hymnes et des prières, soit, beaucoup plus rarement, le 'je' psalmique et sa valeur universelle, qui peut prendre un ton personnel en passant au registre moral, ${ }^{19}$ notamment dans les poèmes pénitenciels. Les appels à Dieu et au Christ qui commentent et ponctuent le récit biblique, les exclamations admiratives ou suppliantes viennent tout naturellement. ${ }^{20}$ Gottschalk d'Orbais, le plus grand poète de l'intériorité, pratique le genre de la prière où l'impératif, adressé à Dieu, se fait lancinant. Dans le superbe $O$ mi custos, ${ }^{21}$ il s'assimile d'abord au fils prodigue, Ego, pater, ille tuus prodigus sum filius (str. 29-36), avant de parler de ce dévoyé à la troisième personne, en l'appelant son frère (str. 37-45) et en implorant de Dieu la même miséricorde que pour lui, mouvement qui montre bien l'assimilation aux misères du fils prodigue, alternant avec la distanciation envers la figure biblique qui a reçu le pardon que lui-même implore encore. Puis Gottschalk passe à Lazare (str. 48-57), en implorant, second Lazare, d'être sauvé comme lui, avec une insistance dans l'objurgation qui réitère, dramatiquement, le moment du miracle : Clama, clama, Iesu Christe, voce magna, domine:/ "Prodi foras, tumulate, veni foras, Lazare, / Exi, exi, ac procede iam mortis de carcere». L'unique impératif de la Bible est multiplié par cinq, enchassé dans la longue série des impératifs de la prière, tentative frénétique de relier le plus étroitement possible le 'je' de l'orant à l'interlocuteur silencieux dont il vocifère la réponse qu'il sollicite. Les deux séries d'impératifs se croisent. La souffrance de Gottschalk s’identifie à la déréliction de Lazare. Il ne s'agit plus ici de paraphrase, mais d'appropriation.

Mais l'apostrophe à l'ennemi, au méchant, qui est l'inverse de la prière, l'exécration au lieu de la louange, est plus original. On le voit mis en œuvre à l'encontre de Judas, assez rarement, mais surtout à l'encontre d'Hérode dans différentes pièces célébrant les saints Innocents, avec toutes les nuances de l'indignation à la compassion. C'est un des lieux privilégiés de l'impératif narratif : on encourage le personnage négatif, par antiphrase. L'impératif, qui sert de supplication vis-à-vis de la divinité, se fait à l'inverse ironique : on encourage l'action perverse parce qu'elle sera sans effet ou aura des effets contraires, en un mouvement emprunté à Prudence, qui le met dans la bouche d'Eulalie : Age Ergo age, tortor, adure, seca ${ }^{22}$.

19 Ibid., p. 232.

20 Les prières liturgiques reposent parfois sur le procédé de transformation d'un texte biblique en imploration par le replacement d'un indicatif par un impératif, ce qui réactualise en demandant pour le présent une intervention divine semblable à celles du passé. Voir Colette et Iversen (2014) 78. 21 Corpus (2007) 315-334.

22 PLAC II 246, str. 7 de Carmina psallere voce lyra, Raban Maur dub. Sauf le cinquième vers, presque toute la strophe est empruntée à Prudence, Cathemerinon 3.91-94. La strophe précédente est également reprise des v. 86-89, seul le cinquième vers étant différent. L'auteur a ainsi calqué trois strophes de Sedulius, puis deux de Prudence ; le défi d'Eulalie à l'empereur Maximien se transforme avec peu de retouches en défi à Hérode. Je remercie F.E. Consolino d'avoir attiré mon attention sur cet emprunt, non identifié par l'éditeur Karl Strecker. 
A côté des impératifs, les interrogations sont une autre façon de dynamiser le propos. Les questions peuvent être un moyen de structuration. Ainsi dans le $D e$ laude Dei alphabétique, Ante saecula et tempora, sont énumérés des miracles bibliques, sur le schéma : Qui a fait cela ? Réponse par le refrain : Benedictus dominus / Christus Dei filius ${ }^{23}$. Le poème joue à fond de l'insistance du refrain, du schéma question-réponse, pour une mise en série (non historique, comme on l'a déjà fait remarquer, puisque par exemple l'expulsion d'Adam du Paradis, str. 21, vient après la lamentation de Rachel sur les Innocents, et la résurrection du fils de la veuve, str. 14, juste après l'arche de Noé). La force du poème ne vient pas de l'insistance sur des détails pathétiques, mais de la convergence dans la direction, tendue vers le salut des hommes par le Christ. Le procédé, question et réponse unique, par accumulation, prend une force non pathétique, mais dramatique par l'intensité du sujet et la force du refrain.

La Bible comporte beaucoup de discours directs. Les paroles du Christ en particulier sont souvent reprises et paraphrasées, même dans les pièces qui ne pratiquent pas généralement ce procédé. Ainsi dans le récit 'De resurrectione Christi', Venit tristis ad Pilatum ${ }^{24}$, qui n'insiste pas sur le pathétique et n'interpelle pas l'auditeur, seules les paroles du Christ sont paraphrasées : Ego sum, nolite flere, carnem meam cernite ... Ego vos, sicut me pater, mitto sicut miserat ... et à Thomas : Mitte manum, tange latus, sis fidelis, Didime : / Felix erit qui viderit et credere potuit (str. 10, 17, 22). Le discours direct y est à peu près le seul procédé dramatisant.

Le discours direct est le plus souvent justifié par le modèle biblique. Mais l'embellissement poétique permet de développer son usage. Ainsi le Pseudo-Paulin, sur Noël, Gloriam deo in excelsis hodie, ${ }^{25}$ présente beaucoup plus de discours directs que dans Matthieu et Luc (str. 15, 23, 25, les deux dernières dans la bouche d'Hérode qui va être violemment pris à partie). Cette pièce, riche par ailleurs de tous les procédés d'interpellations ( $5+1$ str., $26-30$ et 36 , à Hérode, 4 strophes aux Innocents, 38-41) représente parfaitement l'immédiateté dramatique de Paulin d'après Stella, son 'style sentimental'. ${ }^{26}$ En ce sens Paulin est plus proche des rythmes du VIII $^{\text {e }}$ siècle qui lui sont quasi contemporains que de l'intellectualité d'un Florus.

Parfois les paroles sont transformées, généralement dans le sens d'une appropriation de la situation biblique au poète ou à l'auditeur, ainsi les paroles du bon larron dans Surrexit Christus :

23 PLAC IV 524-526.

24 PLAC IV 531.

25 PLAC I 144.

26 Stella (1993) 359 ; Corpus (2007) 206. 
Memora, domine, me peccatorem hominem, qui non sum dignus ego tecum loquere, sed propter meam tantam neglegentiam precor te, Iesus, dona indulgentiam. ${ }^{27}$

Paroles qui pourraient servir à n'importe quel pécheur, et ne reflètent guère les paroles du larron de Lc 23.42 (elles ont peut-être été rapportées, la strophe ayant un vers de trop). On a ici une identification au personnage biblique, comme chez Gottschalk, mais beaucoup moins forte.

Le choix de privilégier tel ou tel locuteur est aussi significatif. Homo quidam erat dives, sur la parabole du mauvais riche et du pauvre Lazare, ${ }^{28}$ Lc $16.23-31$, passe au discours indirect les discours du riche, mais transpose en discours direct les deux réponses d'Abraham le patriarche, qui expriment directement la pensée du Christ.

L'histoire de Joseph, fort dramatique en soi, était spécialement riche en discours, qui ont orienté les réélaborations. Ainsi Audite mirabilia, titré 'De Joseph"29, a six strophes de discours directs. Sur le même thème narratif, Paulin d'Aquilée, Tertio in flore, 'De Jacob et Joseph', ${ }^{30}$ présente d'autres discours directs que la Bible. S'il simplifie les épisodes de l'indignation des frères au récit des rêves de Joseph en ne gardant que les rêves eux-mêmes, et saute les discours directs d'envoi de Joseph par Jacob et une partie des conciliabules entre les frères, en gommant en particulier le rôle de Ruben, il ajoute quatre strophes de lamentation de Jacob (str. 15-18). Puis, sautant l'épisode de la femme de Putiphar, il simplifie également les discours en ne conservant que les rêves et leur interprétation. Dans tous les échanges de la reconnaissance de Joseph et de ses frères, qu'il est obligé d'abréger, il ne garde que les plus émouvants et les plus forts, faisant montre d'un goût raffiné. Le passage ajouté est un planctus : c'est justement vers cette époque que naît le genre. Abélard fera de même, bien plus tard, un Planctus Iacob super filios suos, et les lamentations de Jacob seront abondamment exploitées au XII ${ }^{\mathrm{e}}$ siècle, avec toutes les ressources du pathos. Les poèmes pénitentiels, d'ailleurs, sont l'équivalent de planctus à la première personne : ils ouvrent la voie à la poétique de la plainte. Il est d'ailleurs probable que tous les discours directs, d'abord adaptés de la Bible, puis développés, que l'on rencontre à cette époque, et qui ajoutent à la vivacité des récits bibliques, sont une étape qui accompagne la formation des tropes dialogués aux origines des représentations sacrées.

27 PLAC II 254, str. 6.

28 Dans la version la plus complète, version A, Corpus (2007) 276-278. Plus exactement, il transforme en un vers entier, Filium eum nuncupavit, quod esse debuerat, le vocatif Fili de sa source, ce qui est un début de glose, avant de passer au discours direct ; les autres versions sont plus proches du verset 16, 25. Voir Björkvall - Haug (2003), qui éditent également les deux versions.

29 PLAC IV 640-643, str. 7, 13, 14, 19, 25-26.

30 PLAC IV 462-71; Corpus (2007) 397, commenté par Stella ibid. 347-356. 
Plusieurs discours directs aboutissent aux dialogues. Le dialogue entre Jésus et Judas $^{31}$ en est un parfait exemple, avec ses refrains alternés $O$ Juda, traditor meus et Heu me peccatorem, qui tradidi salvatorem, où les reproches de l'un et la repentance désespérée de l'autre sont conclus par une apostrophe à l'auditoire et à Judas, Et tu, crudeles Iuda, permanes in ardura, bien que l'éditeur Peter Stotz doute de la pertinence de cette dernière apostrophe peut-être plus tardive. Ce dialogue est entièrement tragique. De même que les échanges entre le Christ et les âmes qu'il juge du chapitre 25 de Mathieu sont reprises dans le très ancien poème Apparebit repentina, avec les réponses étonnées des bénis et des damnés : Quando, Christe, pauperem te vidimus ? / Te, rex magne, vel egentem miserati juvimus ? $^{32}$

Le dialogue est l'essentiel également dans des poèmes inspirés non de la Bible mais des apocryphes, lesquels ne sont pas tellement présents dans les poésies bibliques à nous parvenues, mais qui y sont représentés par un superbe résumé du ch. 20 de l'Evangile de Nicodème. Il s'agit de 'De contentione zabuli cum inferno', incipit Audiat celum atque terra, du début du VIII ${ }^{\mathrm{e}}$ siècle. ${ }^{33}$ Il est sous forme de dialogue, comme sa source. Il a pour refrain un appel direct aux auditeurs, Videte principem mortis/ religatum in tormentis, ce qui fait appel à leur imagination visuelle, et en fait des spectateurs. L'Enfer tremble après l'évasion de Lazare, alors que Satan encore sûr de lui croit avoir réduit Jésus et l'avoir envoyé à l'Enfer. Mais celui-ci sait que Satan va devoir combattre Jésus et sera vaincu : Fortis timor me concutit, dixit Infer pavidus : Es cum illo pugnaturus et ab ipso religandus (str. 6). L'apocryphe est prêt à être mis en scènes à la fois hiératiques et déclamatoires, comme le seront plus tard les jeux du théâtre sacré. Mais le poème, entièrement ombreux, avec la sombre jubilation du refrain, 'Voyez le prince de la mort ligoté dans les tourments', a une force dramatique encore plus grande. La mise en forme poétique conserve le dialogue, mais l'épure, néglige les effets dramatiques extérieurs comme les prisonniers qui s'agitent, la voix qui exige que les portes s'ouvrent, les témoignages des différents patriarches. Le poème est concentré sur la terreur croissante des tyrans vaincus, leur déconfiture. Il esquisse tout juste la lâcheté servile de l'Enfer qui, dans l'apocryphe, est tout prêt à se faire le geôlier de Satan puisque le Christ a vaincu. La tension dramatique est perçue entièrement par l'appréhension croissante des forces du mal. Le texte en prose savourait toutes les circonstances de la victoire sur la mort, dans tous les détails, avant et après l'affrontement entre les deux puissances mauvaises. Le poème ne reprend que le désarroi des vaincus, il exalte donc cette victoire en négatif, du point de vue de la défaite.

Indépendamment de leur promesse typologique, ce sont les épisodes les plus pathétiques qui reçoivent le plus souvent une valorisation poétique. Nous avons déjà rencontré à plusieurs reprises l'histoire de Joseph, mais Esther (IV.588) et Judith,

31 Ed. Peter Stotz (2000).

32 PLAC IV 507, r. 17. Str. 11.

33 PLAC IV 636, n LXXXIII. 
illustrant toutes deux un danger mortel écarté dans des circonstances dramatiques grâce à la mort d'un ennemi, sont également présentes, et plus rarement les Macchabées. ${ }^{34}$ Pour le Nouveau Testament, les thèmes favorisés parlent également de mort et de résurrection : d'abord Lazare, objet de pièces séparées, mais aussi les Saints Innocents qui figurent dans presque toutes les pièces sur la Nativité. ${ }^{35}$ L'épisode des Innocents est compris comme un chant de victoire, car non seulement Hérode n'a pas réussi à faire échouer le plan de Dieu et la survie du Christ enfant annonce sa victoire finale, mais encore les enfants morts ont gagné la palme du martyre. La souffrance des mères est à peine citée, à cause du passage de Mathieu, mais on ne s'y arrête guère. Dans son infatuation et sa cruauté, Hérode, traité de renard et de loup par le Ps.-Paulin d'Aquilée, ${ }^{36}$ est le véritable protagoniste de l'épisode ; lié en enfer comme Satan, il apparaît comme une incarnation du Mal. Le drame des enfants suscite une indignation plus forte encore que la compassion, ou plutôt celle-ci ne s'exprime que par l'indignation. ${ }^{37}$ Ce n'est qu'un peu plus tard que le deuil des mères rejoindra le courant des lamentations féminines, à peine perceptible chez Notker de Saint-Gall (Quid tu, virgo mater) ${ }^{38}$, qui se développera dans les jeux liturgiques. ${ }^{39}$ En effet le drame de la bible est à cette époque théologique et peu sentimental (seule la souffrance des sœurs de Lazare entrouvre la possibilité d'attendrissement, chez Paulin).

On peut noter la relative absence des thèmes issus des apocryphes sur l'enfance du Sauveur (Proto-évangile de Jacques, Infantia Salvatoris ou Ps.-Mathieu). Florus, dans ses Gesta Christi, écrits pour une école, fait des allusions aux épisodes de l'enfance, mais de façon assez lointaine et détachée. Ces textes étaient pourtant connus puisqu'on y a des allusions chez certains Pères ou dans les Joca monacho-

34 Chez Walahfrid Strabon, voir Vélez Latorre (2005). Il montre que le combat des Machabées est interprété comme une lutte du Christ contre le mal et le démon.

35 Ainsi PLAC IV 478, r. Nº 7, str. 10 - Ibid. 481, Nº 8, str. 5 - PLAC IV 521 ; 524- 526, str. 20 - Micon de Saint-Riquier, PLAC III 299-300. - Versus Hartmanni de natale Innocentum ad processionem, de Saint-Gall, PLAC IV.1 318. - PLAC IV 351, n IV, chanson à boire pour chaque fête, ms. Reg. lat. 321, de Saint-Julien de Brioude fin $\mathrm{IX}^{\mathrm{e}} \mathrm{s}$.

36 PLAC I 144, n 11 , str. 26-31.

37 Vulpes Herodes, cur cauda dissimulas / predam captare, qui beluino gutture / sanguinem sitis, agni carnes esuris, / lupe crudelis, str. 26 de PLAC I 144, dubia de Paulin, Gloriam Deo in excelsis ; le poème multiplie les imprécations et les adjurations à propos des Innocents, str. 34-41; de même avec une ironie mordante (str. 6, Dux bone, arbiter egregius) Raban, PLAC II 246, voir Stella (1993) 246 et anthologie $\mathrm{n}^{\circ}$ 49. Notker, In Natale Innocentum, PL 131, col.1007-1008, ne parle même pas des mères et insiste surtout sur la sainteté et le pouvoir d'intercession des Saints Innocents. Quant à Micon de Saint-Riquier, PLAC III 299-300, v. 63-72, son indignation rhétorique est dépourvue de la moindre émotion, et sa muse demande ensuite à boire pour sa peine. Mais Micon insiste sur sa propre superficialité : il prétend écrire pour qu'on ne puisse lui reprocher d'être oisif. D’autres poèmes de Saint-Gall (PLAC IV, 318 ; 481) ou d'ailleurs (PLAC IV 524-526, str. 20) sont assez convenus.

38 Stella (1995) 232-234.

39 Voir Colette - Iversen (2014) 271-288, et, pour le rapprochement avec les lamentations féminines mises en musique dans les manuscrits de poètes classiques, Ziolkowski (2007) 195-196. 
rum, et que l'âne et le bœuf apocryphes apparaissent dans les plus anciennes miniatures de la Nativité. Mais ils ne sont pas encore actifs dans la poésie occidentale, et leurs potentialités dramatiques n'intéressent guère. Un rythme sur l'Annonciation, qui va en fait jusqu'au baptême de Jésus et finit avec Zachée (car il est alphabétique), n'en fait pas usage, ${ }^{40}$ non plus que les pièces sur la nativité, ${ }^{41}$ ou à la louange de la Vierge. ${ }^{42}$ Le fait que le Christ se soit fait homme n'entraîne pas à l'époque d'attendrissement, du moins de la part des clercs. Il n'y a pas encore de lamentations de la Vierge, à peine la mention de Siméon lui promettant un glaive dans le cœur, ni de Marie Madeleine. Cette époque s'intéresse peu à l'humanité du Christ sinon au plan dogmatique et théologique, et s'en tient résolument aux textes canoniques. Le seul apocryphe qui a vraiment inspiré les poètes, c'est l'Evangile de Nicodème, à cause de sa tension eschatologique ; on retrouve son influence, même discrète, dans presque tous les poèmes sur la Résurrection ou le Jugement, où Satan grince des dents et les âmes des justes montent au ciel.

La seule conclusion de ce rapide coup d'œil sera une comparaison avec les poèmes analogues de la fin $\mathrm{XI}^{\mathrm{e}}-\mathrm{XII}^{\mathrm{e}}$ siècle, qui ne me permettra que quelques généralités, discutables comme toutes les généralités.

Il me semble que l'accent passe avec le temps de l'eschatologie à la tropologie, avec toutes les nuances à apporter. On rencontre à l'époque carolingienne moins souvent d'épisodes tirés de paraboles : le fils prodigue par exemple apparaît très peu comme source d'inspiration, sauf chez Gottschalk dans $O$ mi custos, alors qu'il est très présent $\mathrm{au} \mathrm{XII}$ e siècle. Le bon Samaritain est absent. Mais sont favorisés les épisodes ayant une valeur eschatologique évidente, comme la parabole du riche et du pauvre Lazare.

Le thème des Innocents nous fait toucher du doigt les caractères propres de l'émotivité de l'époque. Le VIII ${ }^{\text {e }}$ siècle, assimilant dans la punition Hérode à Satan et Judas, est nettement plus vindicatif que les époques postérieures, à partir de Notker le Bègue, où Hérode s'efface ${ }^{43}$; chez Hildegarde au XII' siècle, il n'est plus promis qu'au sommeil de la mort, tandis que la compassion pour les enfants morts prend le dessus.

40 PLAC IV 474.

41 PLAC IV 477, r. 7 A superna caeli parte.

42 PLAC IV 515 Aurora dicta, où toutefois dans la str. 10 Kasta turture voce, vero sermone / se profitentem virum non cognoscere, l'allusion à la tourterelle ne vient pas seulement de Lc 1.34.

43 Notker de Saint-Gall, PL $131 \mathrm{n}^{\circ}$ 6, ne parle que de la rémunération céleste des Innocents, et Hérode qui sert de repoussoir (Herodis ut non fiat socius quisquis) semble très lointain. Dans cette séquence l'accent est sur le présent des orants, sur leur crainte et leur espérance, plus que sur le drame passé. Abélard dans l'Hymnarius paraclitensis est encore dans le droit fil de la tradition qui met en parallèle la tristesse de la mort et la gloire de la récompense, mais, avec une antienne tout aussi liturgique, Hildegarde de Bingen, avec le refrain Sed nubes super eundem sanguinem plangunt, semble intégrer une dimension pathétique de la déploration qui marque une sensibilité nouvelle (éd. Barth-Ritscher-Schmidt, Salzburg 1969, p. 262). 
L'expression de la méthode exégétique me semble un peu moins souvent qu'au $\mathrm{XI}^{\mathrm{e}}-\mathrm{XII}^{\mathrm{e}}$ s. constitutive ; elle reste souvent implicite, sauf chez Paulin où elle est juxtaposée, après le récit, ${ }^{44}$ ou intériorisée, chez Walahfrid, ${ }^{45}$ alors que certains poèmes d'Hildebert sont uniquement fondés sur l'exégèse.

La fascination devant le réservoir de méditation de la Bible est toujours le même, ainsi que le besoin de l'actualiser, donc de l'intérioriser par la rumination poétique. Il me semble que l'insistance est plus à l'eschatologie à la fin de l'époque mérovingienne, notamment dans les rythmes, et plus à la tropologie à l'époque tardive, où ce qui semble l'essentiel, ce sont les mœurs et la foi, dans le présent des fidèles. Sur le plan de la dramatisation, l'arsenal est toujours le même : interpellations aux protagonistes, commentaires, exclamations. Avec le temps, les moyens rhétoriques de plus en plus rodés permettent une surenchère dans la grandiloquence, qu'on pourrait illustrer en plaçant à côté du poème de Paulin d'Aquilée sur Joseph et Jacob, cité plus haut, le même thème traité par Pierre Riga dans son Floridus aspectus : le planctus de Jacob y est un feu d'artifice de 36 vers anaphoriques et baroques, pleins d'apostrophes, d'allitérations et de figures. Le classicisme mesuré de Paulin prend en comparaison toute sa valeur.

\section{Bibliographie}

Björkvall - Haug (2003): Gunilla Björkvall et Andreas Haug , «Rhytmischer Vers : performative Aspekte seiner form zu MGH Poetae IV, 2 n. 35 und n. 88», in: Poetry, 119-148.

Bourgain (2016): Pascale Bourgain, «Mise en texte et ponctuation des rythmes lyriques latins», in: Ponctuer l'œuvre médiévale. Des signes au sens, Etudes réunies par Valérie Fasseur et Cécile Rochelois, Genève (Publications romanes et françaises, 267), 149-181.

Colette - Iversen (2014): Marie-Noël Colette et Gunilla Iversen, La parole chantée, invention poétique et musicale dans le haut Moyen Âge occidental, Turnhout.

Corpus (2007): Corpus rythmorum musicum saec. IV-IX, directed by Francesco Stella I. Songs in non-liturgical sources, I, Lyrics, Firenze (Millennio medievale 72, Testi 18).

PLAC: Poetae latini aevi carolini, Monumenta Germaniae historica

Poesia (2000): Poesia dell'alto Medioevo europeo : Manoscritti, lingua e musica dei ritmi latini. Atti delle euroconferenze per il Corpus dei ritmi latini (IV-IX sec.), Arezzo 1998 e Ravello 1999, a cura di Francesco Stella, Firenze, SISMEL/Ed. del Galluzzo.

Poetry (2003): Poetry of the early medieval Europe : manuscripts, language and music of the rythmical Latin texts, III Euroconference for ... the Corpus of latin rythmical texts 4th-9th Century, a cura di E. D’Angelo e Fr. Stella, Firenze (Millennio medievale 39).

Stella (1993): Francesco Stella, La poesia carolingia latina a tema biblico, Spoleto (Biblioteca di Medioevo latino, 9)

44 La riche terminologie de Paulin d'Aquilée, comportant des expressions qui lui sont propres, a été étudiée par Stella (1993) 343 ; 364. Mais il faut remarquer que la partie exégétique très fournie de Fuit Domini dilectus (p. ex. str. 43) a été négligée par la plus grande partie de la tradition manuscrite, et qu'il n'est pas même sûr qu'elle nous soit parvenue complète (Stella, dans Corpus (2007) 206) : elle est détachable, et non pas intégrée dans le récit.

45 Voir Velez Latorre (2005). 
Stella (1995): La poesia carolingia. Testo latino a fronte, a cura di Fr. Stella, Firenze.

Stella (2001): Francesco Stella, Poesia e teologia. I. L'Occidente latino tra IV e VIII secolo, Milano. Stella (2010) : Francesco Stella, Poesia e teologia. II. Il dibattito carolingio, Milano.

Stotz (2000): Peter Stotz, «Judas Iscarioth als Büsser : zu einer wiedergewonneren rhythmischen Wechselrede zwischen Christus und seinem Verräter», in Poesia (2000), 298-312.

Vélez Latorre (2005): José Manuel Vélez Latorre, «La paràfrasis bìblica en cuatro textos de Walafrido Estrabon », in: Poesía latina medieval (siglos V-XV). Actas del IV Congreso del «Internationales Mittellateinerkomitee', Santiago de Compostela, 2002, ed. Manuel C. Diaz y Diaz \& José M. Diaz de Bustamante, Firenze, 351-356.

Ziolkowski (2007): Jan M. Ziolkowski, Nota bene : Reading Classics and Writing Melodies in the Early Middle Ages, Turnhout (Publications of the Journal of Medieval Latin, 7). 